\title{
BOUNDED INJECTIVITY AND HAAGERUP TENSOR PRODUCT
}

\author{
Mohammad B. Asadi, Alireza Medghalchi and Hamed Nikpey \\ University of Tehran, Tarbiat Moallem University and Shahid Rajaee \\ University, Iran
}

\begin{abstract}
In this paper, we prove that if $V \subseteq B(H)$ is an injective operator system on a separable Hilbert space $H$, then $V \otimes_{h} W$ is b-injective for any operator system $W$ if and only if $V$ is finite dimensional.
\end{abstract}

\section{INTRODUCTION}

Let $B(H)$ be the set of all bounded linear operators on a Hilbert space $H$. Operator spaces are the concrete closed subspaces of $B(H)$ as formulated in $[3]$.

An operator space $V$ is called $b$-injective if there is a $\lambda \geq 1$ such that for given operator spaces $W_{1} \subseteq W_{2}$ any completely bounded map $\varphi_{1}: W_{1} \rightarrow V$ can be extended to a completely bounded map $\varphi_{2}: W_{2} \rightarrow V$ with $\left\|\varphi_{2}\right\|_{c b} \leq$ $\lambda\left\|\varphi_{1}\right\|_{c b}$. An injective operator space $V$ is a b-injective operator space with $\lambda=1$. For more details see $[6,8]$.

An operator space $V \subseteq B(H)$ is called an operator system if $V$ is unital and a self adjoint operator space. It is well known that every injective operator system is a unital $C^{*}$-algebra. In fact, if $V \subseteq B(H)$ is an injective operator system, then there is some completely contractive onto projection $\varphi: B(H) \rightarrow$ $V$. Therefore, $V$ equipped with the following multiplication

$$
\circ: V \times V \rightarrow V \quad \text { s.t } \quad T \circ S:=\varphi(T S)
$$

is a $C^{*}$-algebra $([3$, Theorem 6.1.3]). Therefore, every finite dimensional injective operator system $V$ is in the form of $\oplus_{k=1}^{n} M_{m_{k}}$. Thus for any operator

2010 Mathematics Subject Classification. 47L25, 46L07.

Key words and phrases. Operator system, injective operator space, bounded injective operator space, Haagerup tensor product.

First author was partly supported by a grant from IPM (No. 91470123). 
space $W$,

$$
V \check{\otimes} W \cong \oplus_{k=1}^{n} M_{m_{k}}(W) .
$$

Consequently, if $W$ is an injective operator system then $V \check{\otimes} W$ is an injective operator system, too.

Furthermore, Takesaki in [11] shows that, for every two $C^{*}$-algebras $A$ and $B$, the minimal $C^{*}$-tensor product $A \otimes B$ is injective if and only if $A$ and $B$ are injective and either $A$ or $B$ is finite-dimensional.

The above fact is not necessarily valid in the category of operator spaces, because there exist infinite dimensional injective operator spaces whose minimal tensor product is injective. In fact, let $\delta_{11}$ be the projection in $M_{\infty}$ which is 1 in the first coordinate and zero elsewhere. Thus $K_{1 \times \infty}=$ $M_{1 \times \infty} \cong \delta_{11} M_{\infty}$ is an injective operator space. By [3, Page 177],

$$
K_{1 \times \infty} \check{\otimes} K_{1 \times \infty} \cong K_{1 \times \infty}\left(K_{1 \times \infty}\right) \cong K_{1, \infty \times \infty}=M_{1, \infty \times \infty} .
$$

is again an injective operator space.

Now, in the operator space category the question naturally arises:

whether or not the above-mentioned fact is valid for another cross norm.

In this paper, we focus on the problem considering the Haagerup tensor product. In fact, we prove that if $V \subseteq B(H)$ is an injective operator system on a separable Hilbert space $H$, then $V \otimes_{h} W$ is b-injective for any operator system $W$ if and only if $V$ is finite dimensional.

\section{The Main Theorem}

In this paper, we use the notions of injective and Haagerup tensor products as well as infinite matrices of operator spaces; related to notations and theorems which can be found in $[1,3]$.

Given operator spaces $V$ and $W$ and a linear mapping $\varphi: V \rightarrow W$, for each $n \in \mathbb{N}$, there is a corresponding linear mapping $\varphi_{n}: M_{n}(V) \rightarrow M_{n}(W)$ defined by $\varphi_{n}(T)=\left[\varphi\left(T_{i, j}\right)\right]$ for all $T=\left[T_{i, j}\right] \in M_{n}(V)$. The completely bounded norm of $\varphi$ is defined by

$$
\|\varphi\|_{c b}=\sup \left\{\left\|\varphi_{n}\right\|: n \in \mathbb{N}\right\} .
$$

It is said that $\varphi$ is completely bounded (respectively, completely contractive) if $\|\varphi\|_{c b}<\infty$ (respectively, $\|\varphi\|_{c b} \leq 1$ ). We say that the operator spaces $V$ and $W$ are completely isometrically isomorphic if there is an onto linear map $\varphi: V \rightarrow W$ such that each mapping $\varphi_{n}: M_{n}(V) \rightarrow M_{n}(W)$ is an isometry. This notion is indicated by $V \cong W$. If $\varphi: V \rightarrow W$ is a completely bounded linear bijection and its inverse is completely bounded, then we say $\varphi$ is a completely isomorphism. In this case, we say that $V$ and $W$ are completely isomorphic and write $V \simeq W$. It is well known that the same dimensional operator spaces are completely isomorphic.

Let $V$ and $W$ be $\lambda_{V}$ - and $\lambda_{W}$-injective operator spaces, respectively. Then $V \oplus W$ is a $\max \left\{\lambda_{V}, \lambda_{W}\right\}$-injective operator space. Also, if $Z$ is an operator 
subspace of $V$ and there is a completely bounded onto projection $\varphi: V \rightarrow Z$, then $Z$ is a $\lambda_{V}\|\varphi\|_{c b}$-injective operator space.

LEMMA 2.1. Let $V$ and $W$ be completely isomorphic operator spaces. Then $V$ is a b-injective operator space if and only if $W$ is a b-injective operator space.

Proof. We assume that $V$ is a $\lambda$-injective operator space for some $\lambda \geq 1$, and also $\varphi: W \rightarrow V$ is a completely isomorphic mapping. Let $Z_{1}, Z_{2}$ be two operator spaces satisfying $Z_{1} \subseteq Z_{2}$ and $\phi: Z_{1} \rightarrow W$ be a completely bounded map. Thus $\varphi \circ \phi: Z_{1} \rightarrow V$ is a completely bounded map, and so there is a completely bounded map $\psi: Z_{2} \rightarrow V$ extension for $\varphi \circ \phi$ with $\|\psi\|_{c b} \leq \lambda\|\varphi \circ \phi\|_{c b}$. Obviously $\varphi^{-1} \circ \psi: Z_{2} \rightarrow W$ is a completely bounded extension map for $\phi$ such that

$$
\left\|\varphi^{-1} \circ \psi\right\|_{c b} \leq\left\|\varphi^{-1}\right\|_{c b}\|\psi\|_{c b} \leq \lambda\left\|\varphi^{-1}\right\|_{c b}\|\varphi \circ \phi\|_{c b} \leq \lambda\left\|\varphi^{-1}\right\|_{c b}\|\varphi\|_{c b}\|\phi\|_{c b} .
$$

Thus $W$ is a $\lambda\|\varphi\|_{c b}\left\|\varphi^{-1}\right\|_{c b}$-injective operator space.

THEOREM 2.2. $c_{\circ}$ is not a b-injective operator space.

Proof. Assume, to reach a contradiction, that $c_{\circ}$ is a $\lambda$-injective operator space for some $\lambda \geq 1$. This assumption implies that $c_{\circ}$ is a $\lambda$-injective Banach space, too. In fact, let $E$ and $F$ be Banach spaces, $E \subseteq F$ and $\varphi: E \rightarrow c_{\circ}$ be a bounded linear map. If the Banach spaces $E$ and $F$ endowed with the MIN operator space structure, respectively, then we have $\|\varphi\|=\|\varphi\|_{c b}$. And also, by the assumption, we can extend $\varphi$ to a completely bounded map $\psi:$ MIN $F \rightarrow c_{\circ}$ such that $\|\psi\|_{c b} \leq \lambda\|\varphi\|_{c b}$, and so $\|\psi\| \leq \lambda\|\varphi\|$.

Therefore $c_{\circ}$ is a b-injective Banach space, and so $c_{\circ}$ has a subspace isomorphic to $\ell^{\infty}([8,9])$. On the other hand, the Banach space $c_{\circ}$ is separable, but that $\ell^{\infty}$ is not separable. This is a contradiction.

THEOREM 2.3. Let $V \subseteq B(H)$ be an injective operator system on a separable Hilbert space $H$. Then $V \otimes_{h} W$ is b-injective for all injective operator space $W$ if and only if $V$ is finite dimensional.

Proof. $(\Leftarrow)$ Assume that $V$ is a finite dimensional operator system with $\operatorname{dim} V=n$. Then $V$ is completely isomorphic to the injective column Hilbert space $M_{n, 1}(\mathbb{C})$, ([3, Corollary 2.2.5]). Then by [3, Proposition 9.3.1], we have

$$
V \otimes_{h} W \simeq M_{n, 1}(\mathbb{C}) \otimes_{h} W \cong M_{n, 1}(\mathbb{C}) \otimes \check{W} \cong M_{n, 1}(W) .
$$

Now it is clear that, the injectivity of $W$ implies the injectivity of $M_{n, 1}(W)$. Hence $V \otimes_{h} W$ is b-injective.

$(\Rightarrow)$ Assume that $V$ is an infinite dimensional injective operator system on a separable Hilbert space $H$. By [7], $V$ is completely isomorphic to $\ell^{\infty}$ or $M_{\infty}$

CASE 1) $V \simeq \ell_{\infty}$ : By the assumption of the theorem, $V \otimes_{h} M_{\infty}$ is a b-injective operator space. Thus, by Lemma $1, \ell_{\infty} \otimes_{h} M_{\infty} \simeq V \otimes_{h} M_{\infty}$ is a 
b-injective operator space, too. We can assume that the injective row Hilbert space $K_{1 \times \infty}=M_{1 \times \infty}\left(\cong \delta_{11} M_{\infty}\right)$ is an operator subspace of $M_{\infty}$. Thus, there is some completely contractive onto projection $\varphi^{\prime}: M_{\infty} \rightarrow K_{1 \times \infty}$. By the [3, Proposition 9.2.5],

$$
I \otimes \varphi^{\prime}: \ell^{\infty} \otimes_{h} M_{\infty} \rightarrow \ell^{\infty} \otimes_{h} K_{1 \times \infty}
$$

is a completely contractive and onto projection. Therefore, $\ell^{\infty} \otimes_{h} K_{1 \times \infty}$ is a b-injective operator space. By [3, Page 177 and Proposition 9.3.1], we have

$$
K_{1 \times \infty}\left(\ell^{\infty}\right) \cong \ell^{\infty} \check{\otimes} K_{1 \times \infty} \cong \ell^{\infty} \otimes_{h} K_{1 \times \infty} .
$$

Therefore, by Lemma 1 , there exists some $\lambda \geq 1$ such that $K_{1 \times \infty}\left(\ell^{\infty}\right)$ is a $\lambda$-injective operator space. Let $\delta_{n} \in \ell^{\infty}$ be the natural projection for each $n \in \mathbb{N}$, and $\left(\alpha_{i}\right)_{i} \in c_{\circ}$. We have $\sup _{i}\left|\alpha_{i}\right|<\infty$. Then, for each $n \in \mathbb{N}$

$$
\left\|\left[\begin{array}{llll}
\alpha_{1} \delta_{1} & \alpha_{2} \delta_{2} & \cdots & \alpha_{n} \delta_{n}
\end{array}\right]\right\|=\left\|\sum_{i=1}^{n}\left|\alpha_{i}\right|^{2} \delta_{i}\right\|_{\infty}^{1 / 2}=\max _{1 \leq i \leq n}\left|\alpha_{i}\right| \leq \sup _{i}\left|\alpha_{i}\right|<\infty .
$$

Thus, by definition of $M_{1 \times \infty}\left(\ell^{\infty}\right)$ (see [3], Section 10), we have

$$
u=\left[\begin{array}{lll}
\alpha_{1} \delta_{1} & \alpha_{2} \delta_{2} & \cdots
\end{array}\right] \in M_{1 \times \infty}\left(\ell^{\infty}\right) .
$$

For any $\varepsilon>0$, there is some $n \in \mathbb{N}$ such that $\left|\alpha_{i}\right| \leq \varepsilon$ for each $i \geq n$. For each $m \geq n$, we define

$$
u_{m}=\left[\begin{array}{lllll}
\alpha_{1} \delta_{1} & \cdots & \alpha_{m} \delta_{m} & 0 & \cdots
\end{array}\right] .
$$

We have

$$
\begin{aligned}
& \left\|u-u_{m}\right\|=\left[\begin{array}{llllll}
0 & \cdots & 0 & \alpha_{m+1} \delta_{m+1} & \alpha_{m+2} \delta_{m+2} & \cdots
\end{array}\right] \| \\
& =\sup _{p \geq m+1}\left\|\sum_{i=m+1}^{p}\left|\alpha_{i}\right|^{2} \delta_{i}\right\|_{\infty}^{1 / 2} \\
& =\sup _{p \geq m+1}\left\{\max _{m+1 \leq i \leq p}\left|\alpha_{i}\right|\right\} \leq \varepsilon .
\end{aligned}
$$

Thus, by definition of $K_{1 \times \infty}\left(\ell^{\infty}\right)$, we have $u \in K_{1 \times \infty}\left(\ell^{\infty}\right)$. Therefore,

$$
\varphi: c_{\circ} \rightarrow K_{1 \times \infty}\left(\ell^{\infty}\right):\left(\alpha_{i}\right)_{i} \mapsto\left[\begin{array}{lll}
\alpha_{1} \delta_{1} & \alpha_{2} \delta_{2} & \cdots
\end{array}\right]
$$

is a completely isometric embedding. Now, we consider $\left[\begin{array}{llll}f_{1} & f_{2} & \cdots\end{array}\right] \in$ $K_{1 \times \infty}\left(\ell^{\infty}\right)$. Thus for any $\varepsilon>0$ there is some $n \in \mathbb{N}$ such that

$$
\left\|\left[\begin{array}{llllll}
0 & \cdots & 0 & f_{n} & f_{n+1} & \cdots
\end{array}\right]\right\|=\sup _{p \geq n}\left\|\sum_{i=n}^{p}\left|f_{i}\right|^{2}\right\|_{\infty}^{1 / 2} \leq \varepsilon .
$$

Then for any $p \geq n$ we have $\left\|f_{p}\right\|_{\infty} \leq \varepsilon$. Hence

$$
\psi: K_{1 \times \infty}\left(\ell^{\infty}\right) \rightarrow c_{\circ}:\left[\begin{array}{lll}
f_{1} & f_{2} & \cdots
\end{array}\right] \mapsto\left(f_{k}(k)\right)_{k}
$$

is a completely contractive onto mapping such that $\psi \circ \varphi=i d$.

Let $W_{1}, W_{2}$ be two operator spaces satisfying $W_{1} \subseteq W_{2}$, and $\Phi_{1}: W_{1} \rightarrow$ $c_{\circ}$ be a completely bounded mapping. Then $\varphi \circ \Phi_{1}: W_{1} \rightarrow K_{1 \times \infty}\left(\ell^{\infty}\right)$ is a 
completely bounded map. Since $K_{1 \times \infty}\left(\ell^{\infty}\right)$ is a $\lambda$-injective operator space, for $\varphi \circ \Phi_{1}$ there exists a completely bounded extension $\Phi_{2}: W_{2} \rightarrow K_{1 \times \infty}\left(\ell^{\infty}\right)$, where $\left\|\Phi_{2}\right\|_{c b} \leq \lambda\left\|\varphi \circ \Phi_{1}\right\|_{c b}$. Obviously $\psi \circ \Phi_{2}: W_{2} \rightarrow c_{\circ}$ is a completely bounded extension of $\Phi_{1}$ such that

$$
\left\|\psi \circ \Phi_{2}\right\|_{c b} \leq\left\|\Phi_{2}\right\|_{c b} \leq \lambda\left\|\varphi \circ \Phi_{1}\right\|_{c b} \leq \lambda\left\|\Phi_{1}\right\|_{c b} .
$$

Therefore, $c_{\circ}$ is b-injective, and this is a contradiction.

CASE 2) $V \simeq M_{\infty}$ : Therefore $M_{\infty} \otimes_{h} M_{\infty}$ is a b-injective operator space. Also, $\ell^{\infty}$ and $K_{1 \times \infty}$ are injective operator subspaces of $M_{\infty}$. Thus, there are completely contractive onto projections

$$
\varphi: M_{\infty} \rightarrow \ell^{\infty} \text { and } \psi: M_{\infty} \rightarrow K_{1 \times \infty}
$$

Thus, by [3, Proposition 9.2.5],

$$
\varphi \otimes \psi: M_{\infty} \otimes_{h} M_{\infty} \rightarrow \ell^{\infty} \otimes_{h} K_{1 \times \infty}
$$

is a completely contractive and onto projection. Therefore, $\ell^{\infty} \otimes_{h} K_{1 \times \infty}$ is b-injective, too. This, again, leads to a contradiction.

\section{REFERENCES}

1. D. Blecher and Le Merdy, Operator algebras and their modules: an operator space approach, Oxford University Press, Oxford, 2004.

2. D. Blecher and V. I. Paulsen, Multipliers of operator spaces and the injective envelope, Pacific J. Math. 200 (2001), 1-17.

3. E. Effros and Z. J. Ruan, Operator spaces, Oxford University Press, New York, 2000.

4. M. Hamana, Injective envelopes of operator systems, Publ. Res. Inst. Math. Sci. 15 (1979), 773-785.

5. M. Hamana, Injective envelopes of dynamical systems, in: Operator Algebras and Operator Theory, Longman, Harlow, 1992, 69-77.

6. T. Oikhberg, H. P. Rosenthal, Extension properties for the space of compact operators, J. Funct. Anal. 179 (2001), 251-308.

7. A. G. Robertson and S. Wassermann, Completely bounded isomorphism of injective operator systems, Bull. London Math. Soc. 21 (1989), 285-290.

8. H. P. Rosenthal, On complemented and quasi-complemented subspace of quotients of $C(S)$ for Stonian S, Proc. Nat. Acad. Sci. U.S.A. 60 (1968), 1165-1169.

9. H. P. Rosenthal, The complete separable extension property, J. Operator Theory $\mathbf{4 3}$ (2000), $329-374$.

10. Z.-J. Ruan, Injectivity of operator spaces, Trans. Amer. Math. Soc. 315 (1989), 89-104.

11. M. Takesaki, A note on the direct product of operator algebras, Kodai Math. Sem. Rep. 11 (1959), 178-181.

12. G. Wittstock, Extension of completely bounded $C^{*}$-module homomorphisms, in: Operator Algebras and Group Representations, Pitman, Boston, 1984, 238-250. 
M. B. Asadi

School of Mathematics, Statistics and Computer Science

College of Science, University of Tehran

Enghelab Avenue, Tehran

Iran

and

School of Mathematics

Institute for Research in Fundamental Sciences (IPM)

P.O. Box: 19395-5746, Tehran

Iran

E-mail: mb.asadi@khayam.ut.ac.ir

A. Medghalchi

Faculty of Mathematical Sciences and Computer

Kharazmi University

50 Taleghani Avenue, Tehran

Iran

E-mail: a_medghalchi@saba.tmu.ac.ir

H. Nikpey

Faculty of Mathematical Sciences and Computer

Kharazmi University

50 Taleghani Avenue, Tehran

Iran

E-mail: hamednikpey@gmail.com

Received: 21.11.2011. 